\title{
Influence of low temperature annealing on Nickel RTDs designed for heat flux sensing
}

\author{
Youssef Mokadem ${ }^{1}$, Sylvie Bégot ${ }^{1 *}$, François Lanzetta ${ }^{1}$, Jean-Yves Rauch ${ }^{1}$, Aymen Zribi ${ }^{1}$, \\ Virginie Moutarlier ${ }^{2}$, Magali Barthès ${ }^{1}$ \\ ${ }^{1}$ FEMTO-ST Institute UMR 6174, CNRS-University Bourgogne Franche-Comté-ENSMM-UTBM, 2 \\ avenue Jean Moulin, 90000 Belfort France \\ ${ }^{2}$ UTINAM Institute, UMR 6213, CNRS-University Bourgogne Franche-Comté, 16, Route de Gray \\ 25030 BESANCON Cedex France
}

\begin{abstract}
In this paper, we study the influence of annealing on the performance of Resistive Temperature Detectors made from Nickel thin films. The aimed application is heat flux sensing. The substrate is made of Borofloat glass with a Chromium adhesive layer. Several annealing temperatures between $150^{\circ} \mathrm{C}$ and $300^{\circ} \mathrm{C}$ are applied to this assembly. The thin films as deposited and after annealing are analyzed through SEM images. The evolution of the resistance and the temperature coefficient of the sensor are discussed. An annealing temperature is selected that ensures the repeatability of measurements.
\end{abstract}

\section{Introduction}

Resistive Temperature Detectors (RTD) are widely used as sensors in numerous applications. Several metals can be used for the thin film RTDs: platinum, copper or nickel. Nickel has the advantage of being more cost-effective than platinum, therefore it is suitable for wide diffusion sensors or disposable applications as in biomedical sensing [1][2]. Some authors worked on heat flux sensors using thin film nickel RTDs [3][4][5]. In heat flux sensing, the heat flux can be derived from the difference of two temperature measurements. Thus, in this application, any variation in temperature leads to a large discrepancy in the indicated heat flux. Therefore, temperature sensing has to be accurate and reliable, so the properties of the film, resistivity and Temperature Coefficient of Resistance (TCR), have to be stable. One of the method to ensure this stability is to perform an annealing of the film. This method has been widely studied in thin film Platinum [6-10] and more scarcely for Nickel [11].

Annealing leads to different phenomena that happen at different conditions and temperatures: decrease in micro-strain, raise in crystallinity, diffusion and oxidation of the sublayer if any. Therefore, it is useful to gain knowledge on the influence of an annealing process on the resistivity and TCR of a Nickel RTD associated with a sublayer and substrate. In this paper, we focus our work on the influence of low temperature annealing on the characteristics of a Nickel RTD designed for a heat flux sensing application up to $200^{\circ} \mathrm{C}$. The RTD concept, practical design and microfabrication technique are presented in the first section. In the second section, the characterization of the thin films through profilometry and Scanning Electron Microscopy (SEM) is presented. The experimental setup for the 
temperature and resistance measurement is detailed in the following section. Finally, experimental results for the temperature measurement without and with annealing are presented and discussed.

\section{Concept, design and microfabrication of the sensor}

\subsection{Concept}

Gradient heat flux sensors are based on the application of Fourier's law:

$$
q=-\lambda \frac{\Delta T}{e}
$$

where the temperature difference $\Delta T$ is measured across a substrate of known thickness $e$ and conductivity $\lambda$. The thermal resistance of the sensor $e / \lambda$ is usually small in order to avoid disturbance of the thermal environment.

The resistivity $\rho$ of thin metal film can be expressed by Matthiesen's rule [11]:

$$
\rho=\rho_{T}+\rho_{r}
$$

where $\rho_{T}$ is the temperature-dependant term and $\rho_{r}$ is the residual resistivity term. $\rho_{r}$ includes the contribution from surface scattering, impurities, magnetic disorder effects, grain boundaries and intragranular effects. Thus, using thin film metal RTD in a gradient heat flux sensor requires that the variation of resistivity is only linked to the temperature variation and not to uncontrolled variations of the residual resistivity. If not, this variation divided by a small thermal resistance would lead to large heat flux errors. Assuming the resistivity evolves linearly with temperature:

$$
\rho(T)=\rho_{0}\left(1+\alpha\left(T-T_{0}\right)\right)
$$

where $\rho_{0}$ is the resistivity of the material at the reference temperature $T_{0}$. This implies that the resistance of the RTD can be expressed as:

$$
R(T)=R_{0}\left[1+\alpha\left(T-T_{0}\right)\right]
$$

where $R_{0}$ is the resistance at the reference temperature $T_{0}$. The TCR $\alpha$ can be expressed as:

$$
\alpha=\frac{1}{R_{0}} \frac{d R}{d T}=\frac{R(T)-R_{0}}{R_{0}\left(T-T_{0}\right)}
$$

\subsection{Design and fabrication}

The heat flux sensor is made from two RTDs deposited on each side of a Borofloat glass substrate (Figure 1). It was manufactured in the MIMENTO cleanroom at FEMTO-ST institute. A $20 \mathrm{~nm}$ sublayer made of chromium ensures the adhesion between the substrate and the Nickel film. The thickness of the Nickel track is about $220 \mathrm{~nm}$. The total sensor dimensions are $5 \times 5 \times 0.5 \mathrm{~mm}^{3}$, with a sensing area of $2 \times 2 \mathrm{~mm}^{2}$. More details on the sensor can be found in previous works where platinum RTDs were used [12]. The properties of the materials used in the sensor are summarized in Table 1. At the end of the fabrication process, an annealing at different temperatures $\mathrm{T}_{\mathrm{A}}$ from $150^{\circ} \mathrm{C}$ to $300^{\circ} \mathrm{C}$ during $6 \mathrm{~h}$ was applied to the sensors (Figure 2). 


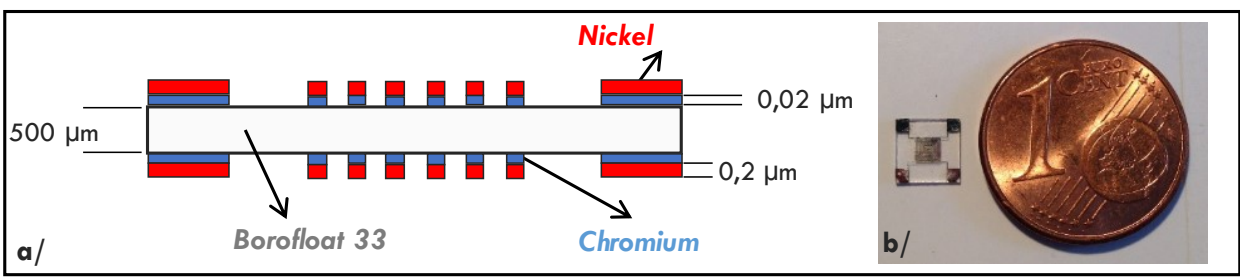

Figure 1 Heat flux sensor scheme (a) and photograph (b)

Table 1. Properties

\begin{tabular}{|l|l|l|l|}
\hline Property & Borofloat Glass & Nickel & Chromium \\
\hline Resistivity $\rho(\Omega \cdot \mathrm{m})$ at $25^{\circ} \mathrm{C}$ & & $6.9 \times 10^{-8}$ & $13.2 \times 10^{-8}$ \\
\hline $\operatorname{TCR} \alpha\left(\mathrm{C}^{-1}\right)$ at $25-500{ }^{\circ} \mathrm{C}$ (bulk) & & 0.0068 & 0.00214 \\
\hline
\end{tabular}

\section{Characterization of the thin films}

The thickness of the Nickel tracks was measured with an accuracy of $0.1 \%$ by a TENCOR profilometer (DEKTAK 150). Two examples of profiles on either side of the substrate are shown in Figure 4. The deposition thickness was of the order of $0.220 \mu \mathrm{m}$ and the track width is $60 \mu \mathrm{m}$ which is in agreement with the design.

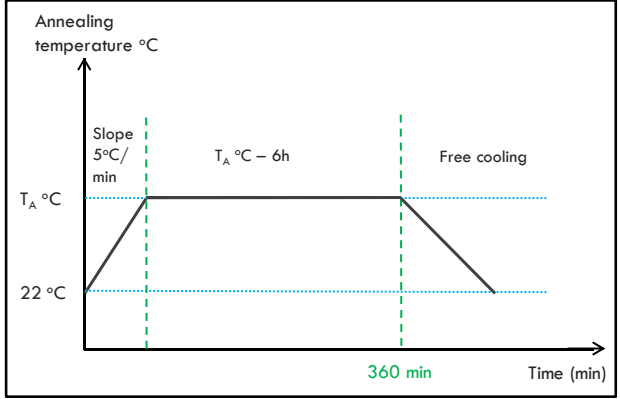

Figure 2 Annealing profile

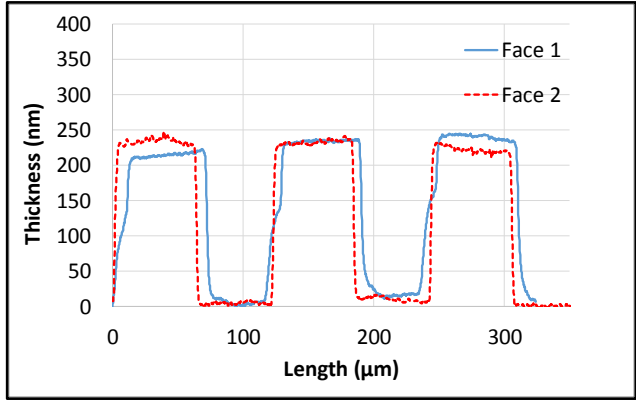

Figure 3 Profiles of the tracks of nickel films.

The characterization of the films was also achieved by SEM. The film surfaces were observed as deposited and after annealing at $200^{\circ} \mathrm{C}$ and $300^{\circ} \mathrm{C}$. We did not find any structural differences as a function of different annealing temperatures (Figure 4).
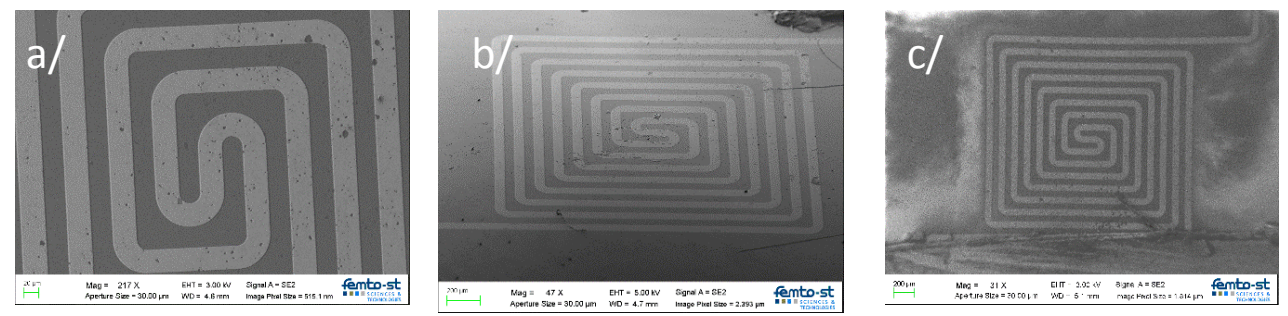

Figure 4. SEM pictures of $\mathrm{Ni} / \mathrm{Cr}$ films a/ as deposited $-\mathrm{b} /$ annealed $200^{\circ} \mathrm{C}-\mathrm{c} /$ annealed $300^{\circ} \mathrm{C}$ 


\section{Experimental results}

\subsection{Temperature calibration bench}

The calibration bench was composed of a portable calibration oven (550 Gemini LRI), a highly accurate Pt 100 platinum reference probe $\left(0.005{ }^{\circ} \mathrm{C}\right)$, a reference thermometer $(\mathrm{PHP}$ 601) (Figure 5a) and two precision $6^{1 / 2}$ digits multimeters Keithley 2100. The RTDs were connected with the 4-wire method with constantan wires. An applied current of $0.1 \mathrm{~mA}$ was applied in order to minimize self-heating. At each temperature step, 50 observations of the resistance value were made. The uncertainty analysis led to a value of $0.14{ }^{\circ} \mathrm{C}$.
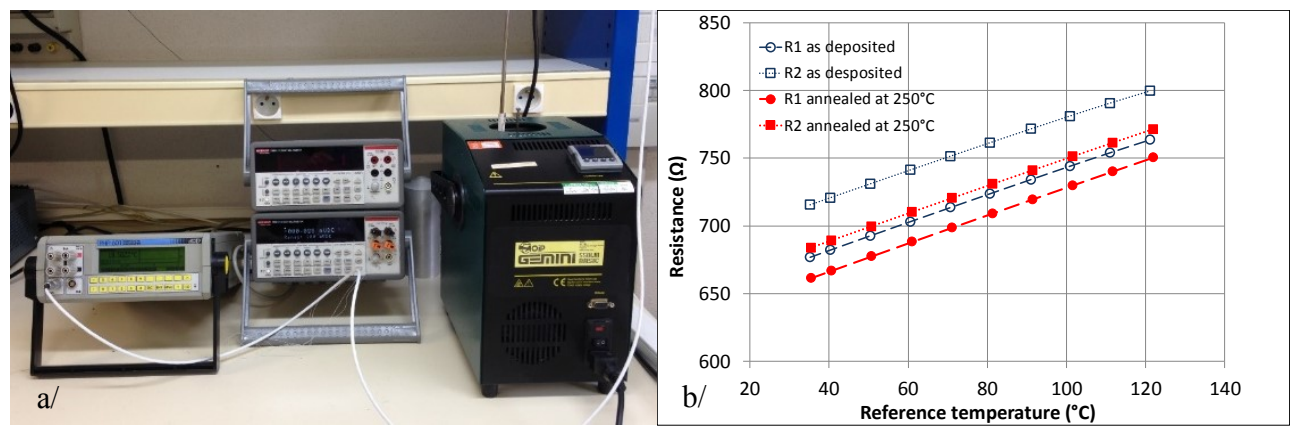

Figure $5 \mathrm{a}$ / Calibration test bench. b/Calibration curves of RTDs as deposited and with annealing

\subsection{Characteristics of the sensor without and with annealing}

\subsubsection{Characteristics of the sensor without annealing}

The initial calibration curves of the nickel RTDs $R_{1}$ and $R_{2}$ as deposited (without annealing) are presented in Figure $5 b$. The difference in the values of resistance $R_{1}$ and $R_{2}$ can be explained by the difference of film deposition from one side to the other one (Figure 2). The observed averaged resistivity of both resistances deduced from the track dimension was $1.31 \times 10^{-7} \Omega$.m at $35^{\circ} \mathrm{C}$ and the average TCR was $0.00143^{\circ} \mathrm{C}^{-1}$ (Table 2). As expected in thin films, we observe an increase in resistivity compared to bulk nickel (Table 1) and a decrease in TCR. As analysed by Johnson [11], the main contribution of this increase is probably due to the raise in grain boundary scattering.

Table 2. TCR as deposited and after annealing at $250^{\circ} \mathrm{C}$

\begin{tabular}{|c|c|c|}
\hline & \multicolumn{2}{|c|}{$\operatorname{TCR}\left({ }^{\circ} \mathbf{C}^{-1}\right)$} \\
\hline Conditions & $\mathrm{R} 1$ & $\mathrm{R} 2$ \\
\hline As deposited & 0.00149 & 0.00138 \\
\hline As deposited : average value & \multicolumn{2}{|c|}{0.00143} \\
\hline After annealing & 0.00156 & 0.00149 \\
\hline After annealing : average value & \multicolumn{2}{|c|}{0.00152} \\
\hline
\end{tabular}

In order to investigate the repeatability of the measurement, 3 successive tests on the same sensor were performed after the calibration (Figure 6 Test1 to Test3). We observed a drift in 
the measurement up to $4.1^{\circ} \mathrm{C}$. Thus, to overcome this problem, different annealing temperatures $T_{A}$ from $150^{\circ} \mathrm{C}$ to $300^{\circ} \mathrm{C}$ as described in $\S 1$ were applied to the sensors.
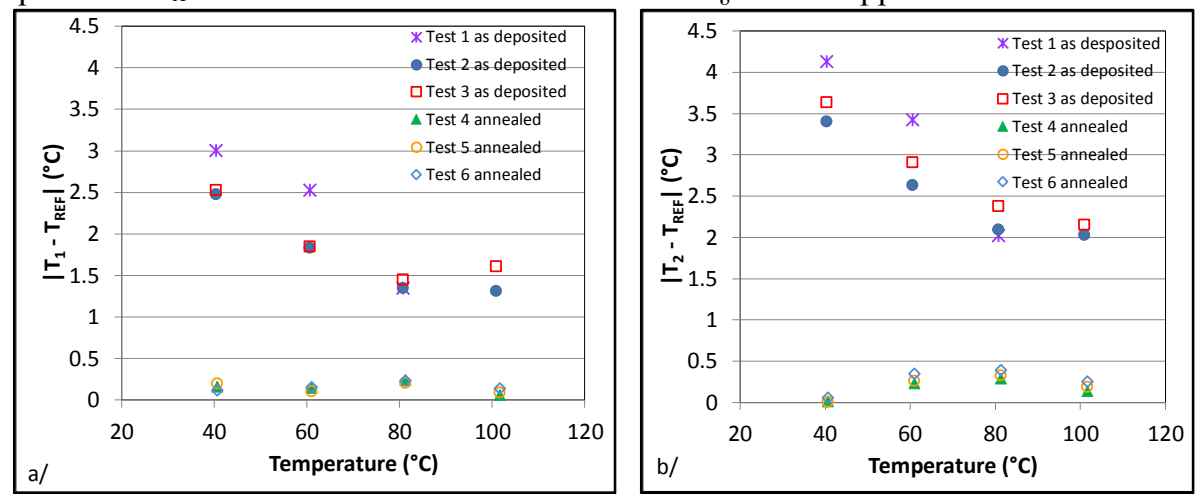

Figure 6 Absolute errors of the RTDs $\left(\mathrm{R}_{1} \mathrm{a} /-\mathrm{R}_{2} \mathrm{~b} /\right)$ as deposited and with annealing at $250^{\circ} \mathrm{C}$

\subsubsection{Characteristics of the sensor with annealing}

The evolution of the resistance at room temperature of the sensor versus the annealing temperature is plotted in Figure 7. We observed a decrease down to $200^{\circ} \mathrm{C}$, then an increase. This phenomenon was also reported by Schmid and Seidel [10] and Zribi et al. [12] on a $\mathrm{Pt} / \mathrm{Ti}$ and $\mathrm{Pt} / \mathrm{Cr}$ film respectively. On the other hand, Johnson only reported a decrease in resistivity on a Ni film without sublayer. Concerning the decrease in resistance observed after annealing at the lowest temperatures, we make the hypothesis that the main contribution is linked to the release of the micro stress induced by the deposition process, as observed by Sreemani and Sen for platinum [9]. A raise in crystallinity due to the annealing can also lead to a decrease in resistivity. Concerning the increase observed above $200^{\circ} \mathrm{C}$, we make the hypothesis that the increase is due to the diffusion and oxidation of the chromium buffer layer into the nickel layer along grain boundaries. This phenomenom was frequently observed for Pt thin films deposited on a sublayer (see for example [8]).

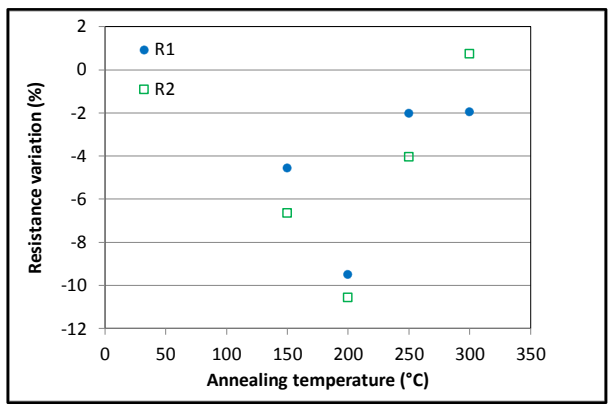

Figure 7 Influence of the annealing temperature on the resistance.

The annealing temperature of $250^{\circ} \mathrm{C}$ was thus chosen in order to benefit from the release of stress in the film. Then, the calibration was performed using the same experimental conditions. We observed a decrease in resistance, thus in resistivity. We also observed a $6 \%$ increase in the average TCR up to $0.00152{ }^{\circ} \mathrm{C}^{-1}$. The absolute errors versus the reference temperature are displayed in Figure 6. We observed that the annealing also led to a high improvement in the repeatability of the results. The absolute errors are reduced to a maximal 
error of $0.44^{\circ} \mathrm{C}$ instead of $4.12^{\circ} \mathrm{C}$ without annealing. Therefore, this choice of annealing temperature led to an improvement of the RTDs.

\section{Conclusion}

In this paper, we have presented the influence of low temperature annealing on nickel RTDs deposited on a glass substrate with a chromium sublayer. The aimed application is heat flux sensing where repeatable and accurate measurement are needed. We applied different annealing temperatures from $150^{\circ} \mathrm{C}$ to $300^{\circ} \mathrm{C}$ for 6 hours and showed that the resistivity decreased down to $200^{\circ} \mathrm{C}$ then increased. The films were analysed though SEM images and no major changes were observed between as deposited and annealed films. We made the hypothesis that the decrease was due to the reduction of stress in the film, and the increase was due to the oxidation and diffusion of the chromium sublayer. Applying a $250^{\circ} \mathrm{C}$ annealing led to repeatable temperature measurements and an increased TCR. The achieved performance seems compliant with the aimed application oh heat flux sensing.

This work has been supported by the EIPHI Graduate School (contract "ANR-17-EURE-0002") and was partly supported by the French RENATECH network and its FEMTO-ST technological facility.

[1] E.J.P. Santos and I.B. Vasconcelos, in 2008 26th International Conference on Microelectronics, (2008).

[2] B. Davaji and C. H. Lee, Biosens Bioelectron, 59,120 ( 2014).

[3] M. Hayashi, A. Sakurai, and S. Aso, Memoirs of the Faculty of Engineering, Kyushu University, 44, 113 (1984).

[4] A.H. Epstein, G.R. Guenette, R.J.G. Norton, and Y. Cao, Rev. Sci. Instrum., 57, no 4, 639 (1986).

[5] H. Mocikat and H. Herwig, Sensors, 9, no.4, 3011(2009).

[6] D. Resnik, J. Kovac, D. Vrtacnik, M. Godec, B. Pecar, and M. Mozek, Thin Solid Films, 639, 64 (2017).

[7] V. Guarnieri, L. Biazi, R. Marchiori, and A. Lago, Biomatter, 4, no. 1, e28822 (2014).

[8] R. Tiggelaar, R. Sanders, A. Groenland, and J. Gardeniers, Sensor Actuat. A: Phys, 152, no.1, (2009).

[9] M. Sreemany and S. Sen, Appl. Surf. Sci., 253, no. 5, 2739 (2006).

[10] U. Schmid and H. Seidel, Thin Solid Films, 516, no. 6, 898 (2008).

[11] B. Johnson, J. Appl. Phys., 67, no. 6, 3018 (1990).

[12] A. Zribi, M. Barthès, S. Bégot, F. Lanzetta, J. Rauch, and V. Moutarlier, Sensor Actuat. A: Phys., 245, 26 (2016). 